\title{
Detecting solar-system gas giant analogs in reflected light with ELTs
}

\author{
Penny D. Sackett \\ Research School of Astronomy and Astrophysics, Australian National University, Mt Stromlo \\ Observatory, Cotter Road, Weston ACT 2611 Australia \\ email: Penny.Sackett@anu.edu.au
}

\begin{abstract}
Cool, extrasolar gas giants similar to Jupiter and Saturn in our own Solar System should be detectable by virtue of the light they reflect in the optical and near-infrared with the next generation of Extremely Large Telescopes (ELTs) equipped with adaptive optics systems. Broad band imaging or very low-resolution spectra should then enable the characterization of the orbit, mass, atmospheric scattering properties, and presence of large rings in these gas giant analogues.
\end{abstract}

Keywords. planets: rings, planetary systems, instrumentation: adaptive optics

\section{Introduction}

Massive planets, believed to be gas giants, have been found orbiting nearby stars at radii ranging from about 0.02 to $5.2 \mathrm{AU} \dagger$. These planets shine in optical and infrared wavelengths in amounts that depend on their mass, shape, distance from their parent star, atmospheric chemical composition, and the presence of clouds. The observed reflected light is modulated throughout one period, and its amplitude depends on the planetparent star separation, and the geometry of the planet, orbit, and any rings relative to the line-of-sight.

To date, reflected light from extrasolar planets has escaped detection, presumably because the brightest ones - those that are closest to their host stars - are confused with the light of their parents, while those at orbital radii just beginning to be resolved by adaptive optics systems are still too faint to be detected by $8 \mathrm{~m}$ - to $10 \mathrm{~m}$-class telescopes. Ground-based, Extremely Large Telescopes (ELTs) with primary mirror diameters of $20 \mathrm{~m}$ to $100 \mathrm{~m}$, however, will be able to detect and characterize these planets in reflected light, if ELT adaptive optics systems can reach the necessary specifications at nearinfrared or optical wavelengths.

In principle, a modulated light signal from the combined planet-star system could be detected without ELTs for very short-period giants without resolving the planet, either by gaining the precision than space could afford (Jenkins \& Doyle (2003), Walker et al. (2003), Green et al. (2003)), or by using the changes in radial velocity (see, e.g., Leigh, Cameron \& Guillot (2003a)) and polarization (Hough et al. (2003)) inherent in the reflected planetary light to distinguish it from host star light. To date, this approach has only produced upper limits on the reflectivity of a few known extrasolar planets, which have been then used to constrain their albedos (Charbonneau et al. (1999), Collier Cameron et al. (2002), Leigh et al. (2003b), Leigh et al. (2003c)).

Examined here are the requirements on ELT high-contrast imaging systems for the resolution and detection of reflected light from cool, gas giants.

$\dagger$ For the most recent statistics, see http://www.obspm.fr/planets 


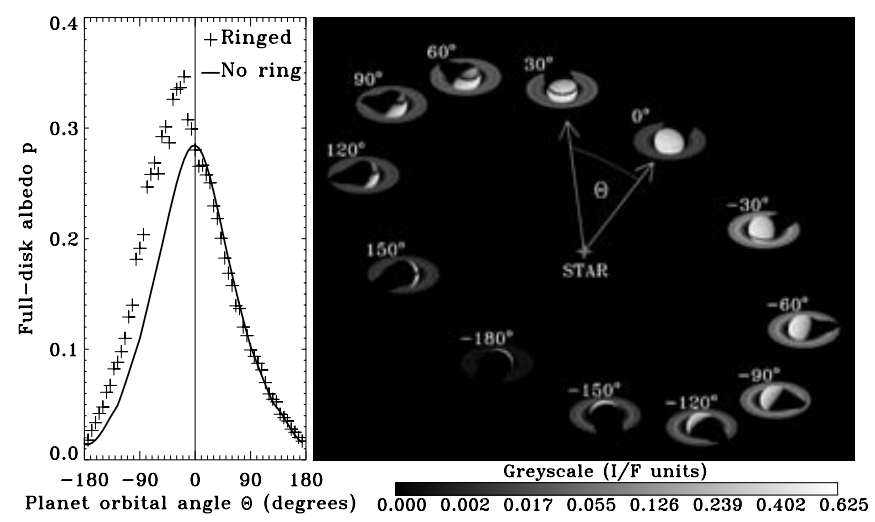

Figure 1. Saturn-like rings contribute significantly to the optical emission from gas giants, and generate asymmetric light curves as a function of orbital phase (from Dyudina et al. (2005)).

\section{Model and model parameters}

In the most general case, the relative geometry of the exoplanetary system and the observer as a function of time must be described by several angles in order to define the amount of reflected light received from the gas giant surface at any time. These include the inclination of the orbit, $i$, and orbital angle, $\Theta$; the phase angle, $\alpha$, can be computed from these two. If the planet has rings, the obliquity of the rings, $\epsilon$, and the azimuth of the observer relative to the rings, $\omega_{r}$, must be considered. If the orbit is significantly eccentric, the argument of pericentre, $\omega$, also enters as an important parameter.

The amount of reflected light collected as a function of time will also depend on the size and shape of the planet and any rings, the scattering properties of the planetary upper atmosphere and of its rings, and the eccentricity of the orbit. Arnold \& Schneider (2004) have studied the effect of ring size, obliquity, and albedo for the case of Lambertian (isotropic) scattering. They find that bright rings may contribute substantially to the total reflected light in the optical, as is the case for Saturn.

Dyudina et al. (2005) expanded on this work by considering oblate planets, realistic (anisotropic) scattering properties for (cold) planets and rings, and orbits characterized by their eccentricity, $e$. They find that the effect of planet oblateness will be small, but rather difficult to disentangle from other effects, especially albedo and orbit geometry. Scattering properties, rings, and eccentricity affect detectability much more strongly. The signature of the rings is generally distinctive because their reflectivity and shadowing creates asymmetric light curves (see Fig. 1).

For planets orbiting at about $1 \mathrm{AU}$ or beyond, thermal emission from the planet itself will peak in the mid-infrared. Cloud cover and hazes are particularly important for the reflected light at optical and near-infrared wavelengths. Marley et al. (1999) showed that the expected total emission from planets could differ dramatically from models of pure thermal emission - even at near-infrared wavelengths - if the possibility of cloud cover was not taken into account. Sudarsky, Burrows \& Pinto (2000) and Seager, Whitney \& Sasselov (2000) considered the effect of different condensates, with different particle sizes, to estimate the behaviour and amplitude of reflected light and polarization curves for very short-period (several day) gas giants, also known as "hot jupiters." Sudarsky, Burrows \& Hubeny (2003) and Sudarsky et al. (2005) went on to compute a suite of 
"self-consistent" model spectra for hot and cool jupiters, respectively, and used these to generate model light curves.

Recognizing the complexity induced by the saturation and covering factor of clouds and hazes, Dyudina et al. (2005) took a different tack, using the actual scattering properties of Jupiter and Saturn as templates for cool, extrasolar gas giants. By fitting published data from the Voyager and Pioneer missions, they generated model phase functions $P\left(\alpha, \mu_{0}, \mu\right)$ separately for Jupiter, Saturn, and the rings of Saturn. Numerically integrating over the full illuminated surface, these phase functions were then transformed into geometric albedos as a function of planet phase, from which light curves for given orbital parameters were created. Jupiter was found to have higher albedos than a Lambertian model would predict both near zero phase, where it exhibits high forward scattering, and at full phase, where its back scattering is pronounced. Saturn's rings exhibit more backward scattering than isotropic rings models at full phase, thus creating more reflection and sharper shadows. The light curves of Dyudina et al. (2005) form the basis of the detectability discussion in the next section.

\section{Detectability}

If the modulated signal of reflected light from a cool gas giant is to be detected in a combined star+planet light curve, the difference between minimum and maximum light must be measurable against the full noise of the bright host star. Fig. 2 displays this difference contrast, $\delta C$, using a shaded scale normalized to a Jovian-sized planet at 5 AU.

If, on the other hand, high resolution imaging can separate the planet from its host, the planet need only be detected against the noise of the background sky and scattered light, in principle allowing its direct detection at all phases and the determination of orbital characteristics.

For the ultra high contrast imaging systems under consideration for next generation systems, the ability to detect directly a faint object close to a bright one is often parameterized by a contrast ratio $C$ as a function of angular separation.

Since the contrast ratios $\delta C$ of Fig. 2 are for the detection of the flux difference between maximum and minimum phase, if $\delta C$ exceeds the contrast levels $C$ planned for high contrast imaging on the ELTs, the planet should be easily detectable at maximum phase. At zero inclination, a planet on a circular orbit has constant (maximum) phase, and so its difference contrast is zero. Its contrast with its host star, however, is about the same as the flux difference contrast at $i=90$, or $\sim 3 \times 10^{-9}$ at 5 AU. Eccentric orbits sometimes bring the planet closer, increasing both the difference contrast and the direct detection contrast at maximum phase. Eccentricities of $e \approx 0.4$, for example, can increase both $C$ and $\delta C$ by factors of $\sim 10$. At higher inclinations, the planet may be detectable over only part of its orbit, but more can be learned about its atmosphere due to the varying illumination (phase).

Contrasts are given for a Jovian at $5 \mathrm{AU}$ in Fig. 2. Scaling to other planet radii $R_{p}$ and orbital semi-major axes $a$ is straightforward: $\delta C$ increases linearly as the square of $R_{p}$ and decreases linearly as the square of $a$, so that a Jupiter-like planet in a circular orbit at $1 \mathrm{AU}$ would require detection a contrast of $\sim 7.5 \times 10^{-8}$. Detectability would be further increased for planets with large rings, which are not included in Fig. 2. 


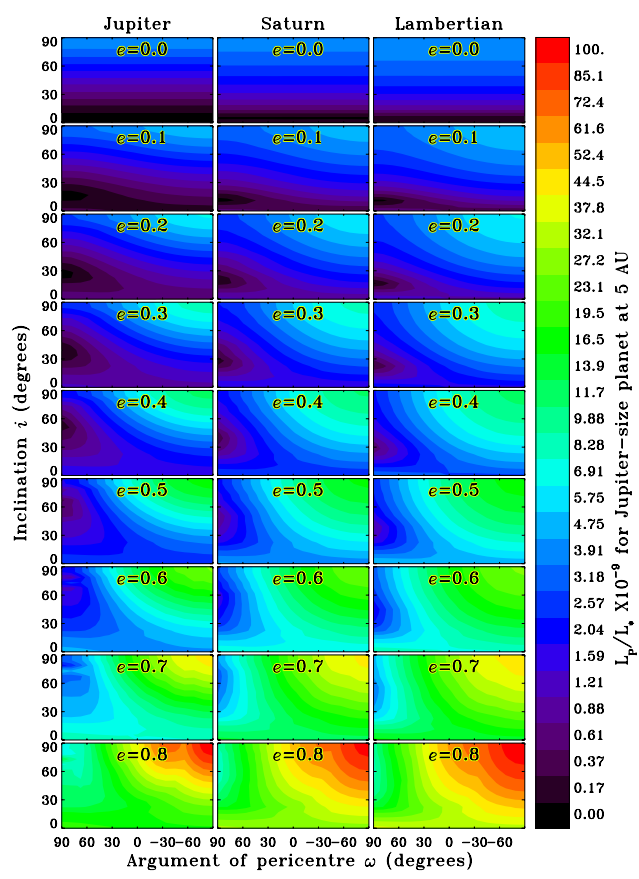

Figure 2. Light curve difference contrasts, $\delta C$, i.e., the difference between minimum and maximum ( $R$ band) flux, normalized by a solar-type host at $5 \mathrm{AU}$, for a Jupiter-sized, ringless, exoplanet. Each subplot describes a different orbital eccentricity $e$ (increasing from top to bottom) and atmospheric scattering properties: Jovian (left column), Saturnian (middle), and Lambertian (right) with surface albedos of 0.82 , to match Saturn. Within each subplot, viewing geometry is described by the inclination $i$ and argument of pericentre, $\omega$ (from Dyudina et al. (2005)).

\section{Implications for ELT}

One of the primary science cases for the largest (100m class) ELT is the direct detection of extrasolar terrestrial planets in reflected light. Such a feat requires contrasts at or below the minimum direct detection levels required for realistic models of cool, gas giants in reflected light. This suggests that gas giant analogs are likely to be detected in reflected light before terrestrial analogs, both because of their higher brightness and their wider separation from the host star which is generating a noisy scattered-light halo in the detector.

State-of-the-art high-contrast imaging systems using coronagraphs and adaptive optics are reviewed elsewhere in these proceedings (see, e.g., contributions from Ellerbroek \& Hubin, Cavarroc et al., and Fusco et al.). We take from the discussion of what is currently being demonstrated in the lab and under consideration for future ELT that a contrast of $10^{-8}$ will be achieved with extreme adaptive optics on ELT. In this case, we would expect gas giants analogs with $a=1 \mathrm{AU}$ (and any $e$ ), and those with $a=5 \mathrm{AU}$ and eccentricities $e \geqslant 0.3$, to be detectable in reflected light - assuming that the planetary systems are not so distant as to require inordinate exposure times, or, as is more likely and troublesome, render planet-star separations too near the inner working angle of the adaptive optics system.

At $1 \mu \mathrm{m}$, this inner working angle (of, say, $3 \lambda / D$ ) would be of order 0.03 arcsec for a $20 \mathrm{~m} \mathrm{ELT}$, and 0.006 arcsec for a $100 \mathrm{~m}$ OWL. Gas giant analogs orbiting at $5 \mathrm{AU}$ will be separated by 0.05 to 0.5 arcsec from parent stars $100 \mathrm{pc}$ and $10 \mathrm{pc}$ distant from us, 
respectively, and thus lie in the working regions of adaptive optics systems of even the smallest ELT. Larger ELT would be able to probe more distant populations of planetary systems by imaging their reflected light directly, and would also be able to perform higher resolution spectroscopy.

Thus, the challenge is not so much primary mirror size, but quality of the imaging optics: regardless of the size of the ELT, contrasts below $1 \times 10^{-8}$ must be achieved for a strict jovian analog ( $e=0$ and $a=5, \mathrm{AU}$; upper left panel of Fig. 2). However, extrasolar gas giant analogs on eccentric orbits, those with semi-major axes between about 1 and $4 \mathrm{AU}$, and those surrounded by substantial ring systems should be detectable with any of the ELT currently being planned - if their adaptive optics systems can reach contrast ratios 10 to 100 times more modest than those required to detect Earth analogs directly.

\section{Conclusions}

- Realistic (anisotropic) scattering results in fainter planets at half phase and at low inclinations

- Eccentricity can increase brightness by factors of several over much of orbit

- Saturn-like rings can increase brightness by factors of 2 or 3 for some geometries

- Assuming optical to near-IR extreme adaptive optics capable of producing contrasts of $10^{-8}$, smaller ELT $(20 \mathrm{~m})$ are capable of detecting gas giant analogs in reflected light in planetary systems $100 \mathrm{pc}$ distant, while $50 \mathrm{~m}$ and larger ELT could probe out to $1 \mathrm{kpc}$

- Gas giant analogs in reflected light are the pathfinders (canaries in the mind shaft) for Earth analog detection projects

\section{Acknowledgements}

I am grateful to the organizers of IAU Symposium 232 for offering me the opportunity to present this overview.

\section{References}

Arnold, L. \& Schneider, J. 2004, A \& A 420, 1153

Collier Cameron, A., Horne, K., Penny, A. \& Leigh, C. 2002, MNRAS 330, 187

Charbonneau, D., Noyes, R.W., Korzennik, S.G., Nisenson, P., Jha, S., Vogt, S.S. \& Kibrick, R.I. 1999, ApJ 522, L145

Dyudina, U., Sackett, P.D., Bayliss, D.D.R., Seager, S., Porco, C.C., Throop, H.B. \& Dones, L. 2005, ApJ, 618, 973

Green, D., Matthews, J., Seager, S. \& Kuschnig, R. 2003, ApJ 597, 590

Hough, J.H., Lucas, P.W., Bailey, J.A. \& Tamura, M. 2003, SPIE 4843, 517

Leigh, C., Cameron, A.C. \& Guillot, T. 2003a, MNRAS 346, 890

Leigh, C., Collier Cameron, A., Horne, K., Penny, A. \& James, D. 2003b, MNRAS 344, 1271

Leigh, C., Collier Cameron, A., Udry, S., Donati, J.-F., Horne, K., James, D. \& Penny, A. 2003c, MNRAS 346, L16

Jenkins, J.M. \& Doyle, L.R. 2003, ApJ 595, 429

Marley, M.S., Gelino, C., Stephens, D., Lunine, J.I. \& Freedman, R. 1999, ApJ 513, 879

Seager, S., Whitney, B.A. \& Sasselov, D.D. 2000, ApJ 540, 504

Sudarsky, D., Burrows, A. \& Hubeny, I. 2003, ApJ 588, 1121

Sudarsky, D., Burrows, A., Hubeny, I. \& Li, A. 2005, ApJ 627, 520

Sudarsky, D., Burrows, A. \& Pinto, P. 2000, ApJ 538, 885

Walker, G., Matthews, J., Kuschnig, R., Johnson, R., Rucinski, S., Pazder, J., Burley, G., Walker, A., Skaret, K., Zee, R., Grocott, S., Carroll, K., Sinclair, P., Sturgeon, D. \& Harron, J. 2003, PASP 115, 1023 


\section{Discussion}

Tolstoy: Why do you say you would need to carry out observations in the optical wavelengths?

SACKETT: We focused on optical wavelengths because we are interested in the reflected light, not the thermal emission. At intermediate wavelengths, the two would be confused, complicating the interpretation of the phase light-curves.

QUIRREnBACH: Have you considered the fact that it is harder to detect a planet when it is closer to the star for your calculations of detectability?

SACKETT: Not in this study, and you are right it needs to be done. I've already made a note to do so in my meeting program. It is also important to compute the amount of time spent in the region of detectability, outside the inner working angle, which depends on telescope diameter.

Herbst: Comment: Another interesting dimension to your plots would be wavelength. After all, Saturn looks radically different at 1, 2, and 3 microns due to atmospheric absorptions and ice absorption in the rings. I bet that this would mostly hurt, but perhaps in some geometries it could help.

SACKETT: Thanks for this comment. We haven't yet looked at the reflectivity and transmission at near-infrared wavelengths. My guess, however, is that the reflectivity will be quite low at those wavelengths for cold gas giants. It would be interesting to attempt to further distinguish rings from the planet body via multi-wavelength discrimination. 